Meta

Journal des traducteurs

Translators' Journal

\title{
Les carences de la traduction littéraire au Canada : des bibliographies et des traditions
}

\section{Gillian Lane-Mercier}

Volume 59, numéro 3, décembre 2014

Traduction et plurilinguisme officiel

Translation and Official Multilingualism

URI : https://id.erudit.org/iderudit/1028655ar

DOI : https://doi.org/10.7202/1028655ar

Aller au sommaire du numéro

Éditeur(s)

Les Presses de l’Université de Montréal

ISSN

0026-0452 (imprimé)

1492-1421 (numérique)

Découvrir la revue

Citer cet article

Lane-Mercier, G. (2014). Les carences de la traduction littéraire au Canada : des bibliographies et des traditions. Meta, 59(3), 517-536.

https://doi.org/10.7202/1028655ar
Résumé de l'article

L'objectif de cet article est double. Dans un premier temps, il vise à mettre au jour deux carences de la traduction littéraire au Canada. La première est d'ordre empirique : il n'existe aucun répertoire complet des oeuvres littéraires canadiennes traduites dans les deux langues officielles. La deuxième est d'ordre théorique : si, en 1977, Philip Stratford a pu constater l'absence d'une tradition de traduction littéraire au Canada, plus de trente ans plus tard, tout porte à croire que la situation a changé. Or, non seulement il n'existe aucune étude d'envergure qui remet en question le constat de Stratford, mais aucun chercheur ne s'est penché sur ce qu'il conviendrait d'entendre par « tradition de traduction littéraire au Canada ». Après avoir exposé ces carences qui, à bien des égards, laissent perplexe en contexte de bilinguisme officiel, il s'agira, dans un deuxième temps, i) de définir l'idée de " tradition "; ii) d'avancer l'hypothèse selon laquelle il existe depuis 1980 plusieurs traditions conflictuelles de traduction littéraire au Canada ; iii) de formuler des questions de méthodologie susceptibles d'en appréhender l'émergence, l'essor et, le cas échéant, l'étiolement ; iv) de souligner l'importance des bibliographies pour la mise au jour de nouvelles pistes analytiques d'ordre tant quantitatif que qualitatif dans le domaine de la traductologie.
Ce document est protégé par la loi sur le droit d'auteur. L’utilisation des services d’Érudit (y compris la reproduction) est assujettie à sa politique d'utilisation que vous pouvez consulter en ligne.

https://apropos.erudit.org/fr/usagers/politique-dutilisation/ 


\title{
Les carences de la traduction littéraire au Canada: des bibliographies et des traditions
}

\author{
GILLIAN LANE-MERCIER \\ Université McGill, Montréal, Québec \\ gillian.lane-mercier@mcgill.ca
}

\begin{abstract}
RÉSUMÉ
L'objectif de cet article est double. Dans un premier temps, il vise à mettre au jour deux carences de la traduction littéraire au Canada. La première est d'ordre empirique: il n'existe aucun répertoire complet des œuvres littéraires canadiennes traduites dans les deux langues officielles. La deuxième est d'ordre théorique: si, en 1977, Philip Stratford a pu constater l'absence d'une tradition de traduction littéraire au Canada, plus de trente ans plus tard, tout porte à croire que la situation a changé. Or, non seulement il n'existe aucune étude d'envergure qui remet en question le constat de Stratford, mais aucun chercheur ne s'est penché sur ce qu'il conviendrait d'entendre par «tradition de traduction littéraire au Canada ». Après avoir exposé ces carences qui, à bien des égards, laissent perplexe en contexte de bilinguisme officiel, il s'agira, dans un deuxième temps, i) de définir l'idée de «tradition »; ii) d'avancer l'hypothèse selon laquelle il existe depuis 1980 plusieurs traditions conflictuelles de traduction littéraire au Canada; iii) de formuler des questions de méthodologie susceptibles d'en appréhender l'émergence, l'essor et, le cas échéant, l'étiolement; iv) de souligner l'importance des bibliographies pour la mise au jour de nouvelles pistes analytiques d'ordre tant quantitatif que qualitatif dans le domaine de la traductologie.
\end{abstract}

\section{ABSTRACT}

The aim of this article is twofold. On the one hand, it proposes to expose two blind spots in literary translation in Canada. The first blind spot is empirical in nature: there is no complete, up to date repertory of the Canadian literary works that have been translated from and into the two official languages. The second is theoretical in nature: in 1977, Philip Stratford remarked on the absence of a tradition of literary translation in Canada. Now, more than thirty years later the situation has likely evolved. However, no major study has called into question Stratford's assertion. Furthermore, no researcher has attempted to define just what is meant by a "tradition of literary translation in Canada." On the other hand, after having explored these blind spots that, from several points of view, are difficult to comprehend in a context of official bilingualism, this article proposes to (i) define the idea of "tradition"; (ii) examine the hypothesis that since 1980 several conflicting traditions of literary translation in Canada have emerged; (iii) formulate a series of methodological questions for the analysis of their emergence, evolution and, perhaps, decline; (iv) underscore the importance of bibliographies in identifying new quantitative and qualitative avenues of research in the area of Translation Studies.

\section{MOTS CLÉS/KEYWORDS}

bibliographies, traditions traductives, traductologie, sociologie de la traduction, traduction littéraire au Canada, recherches quantitatives et qualitatives en traductologie Bibliographies, translation traditions, translation studies, sociology of translation, Canadian literary translation, quantitative and qualitative research in Translation Studies 


\section{Introduction}

Cet article a pour premier objectif de mettre au jour ce qui me paraît être deux carences de la traduction littéraire au Canada. La première carence est d'ordre empirique: il n'existe aucun répertoire complet et à jour des œuvres littéraires canadiennes traduites dans les deux langues officielles. La deuxième est d'ordre davantage théorique: si on a souvent constaté, à juste titre, l'absence d'une tradition de traduction littéraire au Canada (Stratford 1977; Hayne 1983; Ellenwood 1984; Delisle 1987; Simon 1988), tout porte à croire que la situation a changé. En effet, il existe de plus en plus d'études qui nuancent, voire contestent ce constat - que l'on pense aux travaux portant sur l'émergence d'une tradition canadienne de traduction «au féminin » (Lotbinière-Harwood 1991; Simon 1996; von Flotow 1997) - sans toutefois proposer une conception claire de ce qu'il conviendrait d'entendre par «tradition de traduction littéraire au Canada» faute, précisément, de répertoire fiable.

Tout en visant à exposer cette double carence qui peut laisser perplexe en contexte de bilinguisme officiel, à fortiori lorsque la traduction littéraire a pour mission politique d'assurer l'unité nationale, cet article aura pour deuxième objectif i) de définir l'idée de tradition; ii) d'avancer l'hypothèse selon laquelle il existe plusieurs traditions conflictuelles de traduction littéraire au Canada; iii) de formuler des questions de recherche susceptibles d'en appréhender l'émergence, l'essor et, le cas échéant, l'étiolement; iv) de souligner l'urgence de promouvoir, tant sur le plan institutionnel ${ }^{1}$ que dans le cadre d'approches sociologiques de la traduction, des études bibliographiques d'ordre quantitatif et qualitatif. Tout porte à croire que la création, le maintien et l'interprétation d'outils bibliographiques contribueront, audelà de l'analyse dès lors plus rigoureuse de tendances et de traditions traductives, à conférer des assises concrètes et vérifiables à cette affirmation, encore trop spéculative en contexte canadien, de Paul St-Pierre:

Translation makes visible the existence of [the criteria governing the relations between texts and cultures] and in so doing contributes to an awareness of the elements underlying one's own culture, conditioning the definition for one's collective self in terms of (and very often in denial of) another, the other. (1993:63)

De facture programmatique, cet article propose, à partir du constat de ces carences et de la prise en compte de leurs enjeux politiques et idéologiques, l'esquisse d'une approche méthodologique apte non seulement à mettre à l'épreuve le discours fédéraliste sur le statut des langues officielles du Canada en le confrontant à la réalité des pratiques de traduction, mais aussi à reconnaître plusieurs traditions conflictuelles de la traduction littéraire depuis $1980^{2}$.

\section{La carence empirique: pour des études bibliographiques quantitatives}

Commençons donc par cerner la nature et les enjeux de cette première carence que je nomme empirique, soit l'absence d'un répertoire complet et à jour des œuvres littéraires canadiennes traduites de l'anglais au français et du français à l'anglais ${ }^{3}$. Par complet, il convient d'entendre tout d'abord exhaustif. En effet, la banque de données bibliographiques créée par le Conseil des arts du Canada en $1991^{4}$ ne recense que les traductions littéraires subventionnées qui, pour nombreuses qu'elles soient, ne représentent ni la totalité des œuvres effectivement traduites ni celles publiées à 
l'étranger. Ensuite, par complet, il faut entendre systématique au sens où toutes les informations pertinentes s'y retrouvent, dont, par exemple, non seulement le titre, le lieu et l'année de publication des textes source et cible, de même que les noms de l'auteur et du traducteur, mais aussi les cas de coédition, de réimpression, de réédition et de retraduction (voir infra). Enfin, par complet, il importe d'entendre sans erreur, qu'il s'agisse d'informations erronées ou de lacunes (in)volontaires, lesquelles sont malheureusement plus élevées qu'on ne voudrait le croire, y compris dans une base de données aussi réputée que celle de Bibliothèque et archives Canada ${ }^{5}$. Quant à l'impératif d'être à jour, il suffit de se référer à la Bibliographie de livres canadiens traduits de l'anglais au français et du français à l'anglais publiée en 1977 par le comparatiste-traducteur Philip Stratford, ouvrage qui pourrait être considéré comme un modèle du genre en raison, justement, de sa visée d'exhaustivité, mais qui n'a jamais connu de suite. Il en résulte que, depuis une trentaine d'années, nous n'avons pas accès à une vision d'ensemble des œuvres littéraires traduites dans les deux langues officielles - sans parler des très nombreuses langues non officielles, dont l'espagnol, le mandarin et les langues autochtones.

C'est précisément cet état de fait empirique que je cherche de prime abord à creuser en posant la question: pourquoi cette lacune? Car il ne suffit pas de mettre au jour une telle carence, il importe d'en interroger les raisons et les effets. Dans un pays où, comme on l'a maintes fois rappelé depuis l'adoption de la Loi sur les langues officielles en 1969, la traduction institutionnelle fait partie intégrante de nos droits fondamentaux comme de notre identité nationale et de notre quotidien ${ }^{6}$, et où la traduction littéraire est censée permettre une meilleure connaissance de cet "autre» culturel qu'est, selon le cas, l'anglophone ou le francophone, l'absence de données statistiques exhaustives sur la traduction littéraire semble, à bien des égards, frôler l'incompréhensible.

Certes, le fait que la sociologue française Gisèle Sapiro se soit heurtée à des obstacles semblables lorsqu'elle a voulu analyser le marché de la traduction en France entre 1980 et 2006 peut nous consoler jusqu'à un certain point: la non-fiabilité des banques de données à des fins de recherche n'est pas propre au champ canadien. Bien au contraire, elle fait partie des enjeux soulevés par la compilation et l'utilisation des chiffres. Aussi convient-il de relativiser le critère d'exhaustivité en le situant par rapport à des critères d'ordre pragmatique et idéologique. Comme le note Sapiro, "[1] a production des chiffres est une pratique sociale. Dans une perspective sociologique, la question n'est pas seulement "comment utiliser ces données" mais "qui produit quoi et pour qui?" ". Et elle ajoute: "Afin de ne pas tomber dans le fétichisme du chiffre, il faut se donner les moyens de comprendre les conditions de production et la validité des données utilisées» (Sapiro 2008: 46).

\subsection{De la carence empirique à la contradiction idéologique}

Il reste que, pour pertinentes que les mises en garde de Sapiro puissent être, l'absence d'une suite à l'entreprise de Stratford représente, dans un contexte de bilinguisme officiel, une lacune réelle dont il importe de prendre la mesure. Cette lacune me paraît d'autant plus importante qu'elle porte atteinte à la crédibilité, d'une part, du discours fédéraliste dit "pancanadien» dont l'un des objectifs explicites est de promouvoir de manière paritaire la traduction littéraire à l'échelle nationale, et, d'autre part, des 
institutions, fédérales ou non, aptes à véhiculer ce discours. Comme le notait Ray Ellenwood en 1983 - et sa remarque demeure, avec quelques bémols importants, pertinente - :

Canada has a vast machinery for the translation of government documents, technical and financial papers, even advertising copy [...] for the simple reason that the money and the political desire are there. Cultural reports have high priority, while major literary works wait sometimes for years, if they ever get translated at all. These facts represent very clearly an attitude, a sense of values which is reflected every day in our government policies, in the actions of authors and publishers, by the indifference to literary translation shown by reviewers, by the media in general and (not surprisingly), by the public. (1983: 62)

En d'autres termes - et ce sera ma première hypothèse de travail -, l'absence d'un répertoire complet et à jour des traductions littéraires publiées dans les deux langues officielles depuis 1978 pourrait être perçue comme symptomatique, au mieux, d'une indifférence à l'égard de l'«autre» culturel, accompagnée d'un manque de curiosité pour les processus de transfert interculturel concrets qui informent notre identité collective; au pire, d'une contradiction idéologique du bilinguisme officiel qui rend sinon impossible, du moins extrêmement difficile la création d'un tel répertoire. Il s'ensuit que, outre la question : pourquoi cette lacune?, il faut se demander : pourquoi persiste-t-elle ${ }^{7}$ ?

Voici, à mon sens, le véritable problème: d'un côté, le discours fédéraliste insiste, au nom des principes libéraux d'égalité, de citoyenneté indifférenciée, de bilinguisme et d'uninationalisme (voir Maclure 2000: 148-149) ${ }^{8}$, sur l'importance socioculturelle et politique de la traduction en général et de la traduction littéraire en particulier; principes qui sont consacrés depuis 1972 sous la forme de subventions à la traduction littéraire octroyées par le Conseil des arts du Canada (d'une valeur de 1089000 \$CDN en 2011-2012). Mais de l'autre côté, il semblerait que l'on se soucie peu de faciliter (lire: financer) la production d'outils qui permettraient de consigner et, par suite, d'analyser les faits, soit les processus et les produits qui sont partie prenante de la traduction littéraire définie simultanément comme pratique sociale et comme vecteur des échanges culturels. Ce faisant, on court le risque de naturaliser l'espace intranational de circulation des textes traduits en occultant les rapports de force économiques, politiques et culturels sous-jacents, au profit d'une vision harmonieuse de cohabitation pacifique et de transferts interculturels fluides (voir Simon 1997). Pour preuve, on trouve trois formulations récentes du rôle du traducteur sur le site du Conseil des arts, qui reflètent l'idée d'un fédéralisme coopératif, bilatéral et égalitaire entre les deux «peuples fondateurs» - les Français et les Anglais - au cœur du discours politique dominant depuis la création du Dominion du Canada en 1867:

- Il y a d'abord un bon livre. Puis un traducteur, un écrivain ou un éditeur souhaite le traduire.

Soudain, des milliers de lecteurs découvrent l'autre face de la littérature canadienne ${ }^{9}$;

- Les traducteurs soulèvent l'intérêt, l'engouement et la passion des lecteurs ${ }^{10}$;

- Construire des ponts entre le Canada anglais et français, jouer les passeurs de culture, c'est ce que font les traducteurs littéraires dans la plus discrète perfection ${ }^{11}$.

Il me paraît indispensable de mettre en cause cette vision plutôt idéaliste qui, ancrée dans une série de raccourcis somme toute discutables, reconduit explicitement 
la métaphore de la traduction comme pont entre «deux solitudes» largement ignorantes l'une de l'autre forgée dans les années 1960 (voir Lane-Mercier 2011), à laquelle ont adhéré avant tout des traducteurs anglo-canadiens désireux de «faire comprendre» à leurs lecteurs tant la vitalité de la littérature québécoise que les bouleversements sociopolitiques qui secouaient alors le Québec. Ce faisant, il sera possible de rendre visible ce qu'une telle vision passe sous silence, à savoir les textes, les agences, les agents, les contextes et les pratiques traductives réels à l'origine de cette «découverte» et de cet «engouement» pour l'autre, posés comme autant d'évidences qu'il faudrait interroger.

Or, cette remise en cause ne saurait être que partielle tant que l'on ne disposera pas d'un répertoire à jour de données bibliographiques, dont chaque entrée contiendrait dix variables de base:

- pour l'original: auteur(s), titre, éditeur, lieu et date de publication;

- pour la traduction: titre, traducteur(s), éditeur, lieu et date de la publication; auxquelles d'autres variables seraient susceptibles de s'ajouter:

- pour l'original: éditeur, date(s), lieu(x) de réimpression, de réédition, de retraduction; prix littéraire(s); tirage; nombre de comptes rendus;

- pour la traduction: obtention (ou non) d'une subvention; tirage; prix; éditeur, date(s), lieu(x) de réimpression, de réédition, de retraduction; retraducteur(s); nombre de comptes rendus.

Ces variables, sur lesquelles je reviendrai, serviraient de point de départ à des analyses statistiques qui, en fournissant des assises empiriques qu'il importerait par la suite d'interpréter, seraient susceptibles de mettre à l'épreuve les présupposés véhiculés par les énoncés du Conseil des arts, dont surtout le caractère unitaire de «la» littérature canadienne et, par là, la primauté d'une perspective uninationale; l'idée de bon-ententisme et de parité («ponts»); l'impact immédiat («soudain») et assuré de la traduction («des milliers de lecteurs»); l'invisibilité («discrète») du traducteur qui maîtrise son art à la «perfection». Voilà, me semble-t-il, autant de topoï constitutifs du discours fédéraliste sur le bilinguisme officiel et la traduction depuis les années 1960 que le répertoire contribuerait, le cas échéant, à problématiser.

\subsection{Des "valeurs fondamentales"}

Dans le même ordre d'idées, il convient de noter la ténacité de la conception résolument binaire de la société canadienne («l'autre face»; "le Canada anglais et français») qui continue d'informer le discours fédéraliste et ce, en dépit de tentatives récentes de l'atténuer. À cet égard, si trois des neuf valeurs fondamentales sur lesquelles reposent le mandat et les politiques actuels en matière de traduction littéraire du Conseil des arts témoignent de ce que l'on pourrait appeler une volonté de triangulation (l'inclusion d'autres langues de traduction) et d'hétérogénéité (le respect des différences), le rapport binaire, momentanément désamorcé, se recompose à un autre niveau. Aussi peut-on constater dans l'extrait qui suit d'éventuelles tensions entre, d'une part, les deux langues officielles et les langues non officielles (à peine identifiées, les langues autochtones recouvrant plus de 60 langues distinctes); d'autre part, la «perspective nationale» (pancanadienne) fondée sur l'inclusion (l'homogénéité) et les références à la diversité. Le Conseil, comme organisme, 
[e]st convaincu de l'importance d'une perspective nationale concernant les arts, qui enrichit les connaissances du Conseil et de la communauté artistique, favorise des attitudes inclusives des différentes formes d'art et traditions artistiques et assure un leadership à l'échelle nationale et internationale;

[r] especte les langues officielles du Canada, et reconnaît la nécessité de soutenir l'activité artistique professionnelle des Canadiens francophones et anglophones;

[r] especte la diversité régionale au Canada, et reconnaît la nécessité de soutenir l'activité artistique professionnelle partout au pays;

[r]especte l'histoire, les traditions, les langues et les pratiques contemporaines des peuples autochtones et vise à favoriser le développement des artistes et organismes artistiques autochtones;

[r]especte les artistes et organismes artistiques de différentes origines et traditions culturelles et raciales, et vise à faire progresser le travail de ces artistes et organismes artistiques $^{12}$. (Souligné dans le texte)

Dans cette optique, l'établissement d'un répertoire de données bibliographiques exhaustives (on se souviendra que le Conseil ne recense que les œuvres qu'il subventionne) ouvrirait la voie à des analyses d'envergure, jusqu'ici difficilement envisageables, qui permettraient de contextualiser les données que le Conseil met à la disposition du public. On aurait ainsi une meilleure appréhension non seulement de l'impact effectif de son mandat sur l'évolution des processus et des politiques traductionnels au Canada, mais aussi des rapports de force que ce mandat tend à minimiser, selon le principe que ces derniers se laisseraient saisir de prime abord dans les déséquilibres et autres disparités manifestes à même les chiffres bruts, à commencer par le nombre de traductions subventionnées versus le nombre de traductions non subventionnées par année.

Car il y a fort à parier que l'absence d'un répertoire fiable depuis une trentaine d'années n'est peut-être pas le fruit du hasard, à plus forte raison lorsque l'on constate que des projets comparables ont été menés ailleurs: à titre d'exemple, on consultera en ligne la bibliographie TRASNA de la littérature irlandaise en traduction établie par des chercheurs de la Dublin City University, de même que celle de la littérature étrangère en traduction espagnole établie par El Grupo de Investigación Traducción y Lenguajes Especializados rattaché aux universités de Malaga et Pablo de Olavide (Séville) ${ }^{13}$. À ce titre, il y aurait lieu de s'interroger sur la réticence explicite du Conseil de recherches en sciences humaines du Canada, organisme fédéral qui subventionne la recherche universitaire, à financer la création d'outils bibliographiques. Ceci expliquerait, du moins en partie, le fait que l'entreprise de Stratford n'a jamais été poursuivie, les chercheurs ayant eu tendance à établir des bibliographies ou bases de données partielles afin de répondre à des questions de recherche précises sur des thèmes, des genres ou des problématiques spécifiques (voir par exemple Nichols 2001; Haywood 2002; Linteau 1996; Simon 1988; Koustas 1997, 2008; Brisset 1990). Compte tenu, d'une part, de l'éparpillement des sources qu'il s'agirait de confronter et, d'autre part, des conditions de recherche actuelles dans les universités canadiennes, poursuivre l'initiative de Stratford nécessiterait l'allocation de ressources considérables, sur plusieurs années, en vue de l'embauche d'assistants de recherche à qui il incomberait de faire la collecte de données et les analyses statistiques. Force est de constater que de telles ressources n'ont pas encore été octroyées. 
Plutôt que de spéculer sur les raisons, avançons que cette absence a pour effet d'alimenter la vision fédéraliste d'un bilinguisme officiel non problématique, étayé par des processus traductionnels parfaitement bien huilés. Allons plus loin: cette carence entretient l'indifférence et le manque de curiosité à l'égard de la littérature de l'«autre» francophone et anglophone attestés depuis au moins 200 ans, en dépit des efforts déployés par les traducteurs, les éditeurs et le Conseil des arts pour la faire connaître. D’où l'hypothèse avancée plus haut selon laquelle cette carence empirique est symptomatique d'une contradiction de l'idéologie du bilinguisme officiel; d'où, également, l'importance de reprendre en la reformulant l'une des questions posées ci-dessus: quels intérêts politiques et culturels la persistance de cette carence empirique sert-elle et, surtout, que pourrait nous apprendre un répertoire tel celui envisagé ici?

\subsection{De la non-neutralité des chiffres}

Il est vrai que, du point de vue du discours fédéraliste fondé, on l'a dit, sur le principe d'égalité, il peut valoir mieux ne pas tout savoir sur ce qui a été traduit et ce qui ne l'a pas été. Cela permet d'éviter, notamment, les découvertes (du genre: seulement $\mathrm{X} \%$ des œuvres littéraires acadiennes ont été traduites en anglais depuis 1978) susceptibles de heurter telle ou telle communauté culturelle. Citons de nouveau Sapiro: «il faut se donner les moyens de comprendre les conditions de production et la validité des données utilisées" (2008: 46), ce qui revient à confirmer, si besoin est, que les compilations de données empiriques ne sont jamais neutres, y compris celle projetée ici, dont la visée idéologique résiderait dans la révélation des écarts entre la représentation harmonieuse de l'espace traductionnel canadien et la réalité, plus houleuse, des processus de traduction.

Dans cette perspective, il est possible que le caractère lacunaire des principales banques de données consultées - celles du Conseil des arts du Canada, de Bibliothèque et archives Canada et de l'Index Translationum (dont les informations sont fournies par Bibliothèque et archives Canada) - trouve sa validité dans l'image rassurante qu'elles offrent de la présence de l'activité traduisante au Canada et du rôle du gouvernement fédéral, sans que cette activité et ce rôle puissent être comptabilisés et vérifiés au-delà des limites imposées, précisément, par les conditions de production des données recensées.

Posons que, à l'instar des historiens qui visent à sonder les silences de l'histoire, les traductologues ont tout à gagner à se pencher sur les silences (exclusions, omissions, lacunes, erreurs) des bases de données existantes, quelles qu'elles soient. Comme le note Jane Koustas à propos de la traduction d'œuvres québécoises en anglais, la question est de savoir «what has been left out. [...] how is EnglishCanadian's readers' knowledge and understanding of Quebec francophone literature limited by the titles available in translation?» (Koustas 1997: 47). Il faudrait par conséquent disposer des moyens nécessaires pour combler ces blancs - en l'occurrence, créer une liste complète d'œuvres non traduites - afin de procéder à des analyses susceptibles d'identifier, outre l'ensemble des processus, produits, acteurs et agences constitutifs de l'activité traduisante, leurs effets concrets sur les échanges littéraires depuis la fin des années 1970, que ces effets soient redevables à des organismes subventionnaires comme le Conseil des arts, à des maisons d'édition qui cherchent à se positionner sur le marché de la traduction national et/ou international 
grâce à des protocoles éditoriaux portant sur les critères de sélection et de diffusion particuliers, ou encore, bien que plus rarement, à des traducteurs qui s'efforcent de mettre à mal les normes de traduction dominantes. Tout porte à croire que la création d'un répertoire aussi exhaustif que possible fournirait une image à la fois moins «tranquille» et nettement plus complexe - lire: autrement valide - de l'activité traduisante, partant, des rapports réels entre textes et cultures tels qu'ils se nouent et se dénouent depuis la fin des années 1970 dans un pays officiellement bilingue comme le Canada.

Du coup, se profileraient peut-être des traditions de traduction - terme qui reste à définir - encore imperceptibles en raison de l'ampleur des données non répertoriées (ou si dispersées qu'on ne les «voit» pas), qui contribueraient à une compréhension renouvelée du soi et de l'autre, pour reprendre la réflexion de St-Pierre citée en introduction. Car si les compilations bibliographiques, de même que les analyses statistiques qui en découlent, ne sont jamais neutres, c'est justement parce que, en tant que pratiques sociales, elles contiennent en creux leurs conditions de production et de destination et, de ce fait, captent certains aspects du contexte culturel, idéologique et politique dont elles sont tributaires. Dès lors, il conviendrait de s'interroger, sur le plan méthodologique, sur les critères d'établissement et de présentation d'un répertoire complet des œuvres littéraires canadiennes traduites dans les deux langues officielles depuis 1978, sachant que ceux retenus par Stratford auraient besoin d'être réévalués. Ce faisant, il conviendrait également, à la suite de Sapiro (2008) et sur le plan méta-réflexif, de répondre aux questions de savoir «"qui produit quoi [...,] pour qui” [...]» et à quelles fins - questions qui, implicites dans toute recherche bibliographique, néanmoins dépassent le cadre de la présente étude.

\section{La carence théorique: pour des études bibliographiques qualitatives}

La conception à priori pacifique et paritaire des processus de traduction au Canada doit être contestée en raison des angles morts (au sens de ce qui est caché au champ perceptif) qu'elle contient. On l'aura compris : le problème n'est pas d'ordre exclusivement empirique. Si la carence de données empiriques nous empêche de répondre à des questions aussi simples que: quelles œuvres littéraires ont été traduites?, quand?, par qui?, où?, pour qui ?, pourquoi?, cette carence rend également difficiles des études d'envergure de l'activité et du processus traduisants à partir du moment où elles engagent l'un ou l'autre de ces paramètres dans une visée de complétude. Car c'est très exactement sur le plan de la visée de complétude que je me situe: l'absence d'un répertoire exhaustif et à jour des œuvres traduites dans les deux langues officielles impose de facto des limites à quiconque désire analyser les lignes de force et de tension de la traduction littéraire au Canada depuis la fin des années 1970.

Ceci n'est pas un jugement de valeur mais une question de dimensions du corpus primaire. Comme on l'a dit, tout chercheur peut consulter les bases de données existantes ou établir sa propre base à des fins de recherche, de sorte que, dans bon nombre de cas, l'absence d'un répertoire de toutes les traductions d'œuvres littéraires depuis 1978 ne pose aucun problème. Il n'en demeure pas moins que ce dernier représenterait un outil puissant qui permettrait des mises en contexte et des comparaisons à une plus vaste échelle. Il ne s'agit donc pas de dire que tant que les chercheurs n'auront pas accès à une telle source, ils ne pourront pas avancer dans leur réflexion; il faut 
plutôt soutenir qu'à partir du moment où ils y auront accès, ils pourront avancer autrement, voire plus sûrement, tout en formulant de nouvelles questions de recherche à la lumière des analyses statistiques qu'il sera dès lors possible de mener.

Plus précisément, la bibliographie de Stratford a rendu possibles des études percutantes de «la vision qu'on se fait de la littérature canadienne et québécoise via le corpus "littéraire" traduit» (Giguère1983: 48. Voir aussi Ellenwood 1983; 1984; Shouldice 1983; Koustas 1997; 1998; à paraître). L'étude de Giguère, notamment, est on ne peut plus éloquente, qui souligne à propos de la traduction vers l'anglais:

[...] la nette domination des romanciers [...] des années soixante. Les 22 auteurs de cette décennie représentent le tiers de tous les romanciers traduits et leurs œuvres $40 \%$ des titres. [...] mon étude statistique m’a amené à conclure que les années soixante servent de base, [...] de point de référence pour traduire la littérature québécoise. [...] puis le retour en arrière - les années cinquante, quarante et au-delà - s'effectue à partir d'une vision, d'une conception de la littérature québécoise bien ancrée dans les années soixante. (1983: 54-59)

Voici un projet qu'il serait temps de reprendre et de mettre à jour.

Autre exemple: il me semble que l'on ne peut envisager que des réponses partielles aux deux questions, pourtant cruciales, posées par Kathy Mezei dans un article paru en 2003: "At the beginning of this twentieth-first century, how do we stake out a history of literary translation in Canada? [...] Is there a specific Canadian translation experience?» (Mezei 2003: 107; voir aussi Mezei et al. 2014). Si des traductologues étrangers, dont Gentzler (1993), Bassnett (1993) et Munday (2001), ont souligné l'existence d'une «Canadian school of translation» et si les historiens de la traduction littéraire canadiens ont publié des travaux incontournables avec les données à leur disposition (voir Delisle 1987; Godbout 2004; Simon 2006), l’histoire générale de la traduction littéraire au Canada depuis la conquête de la Nouvelle-France par l'Angleterre (1759) reste toujours à écrire, peut-être selon le modèle de l'Histoire des traductions en langue française, dont le premier des quatre tomes est paru en 2012 (Chevrel et al. 2012). Mais c'est la question portant sur «a specific Canadian translation experience» qui sous-tend ma deuxième hypothèse, déjà esquissée ci-dessus, selon laquelle plusieurs traditions de traduction littéraire propres au contexte canadien ont commencé à émerger aux alentours de 1980, soit quelques années à peine après le constat négatif de Stratford (voir infra).

De nouveau, seul le recours à un répertoire exhaustif permettrait à la fois de cerner ces traditions traductives qui, nous le verrons, sont bien plus que de vagues tendances en raison surtout de leur pérennité, et d'en suivre aussi bien l'essor et l'évolution que les interactions. Cela rendrait possibles la conceptualisation et la comparaison de la spécificité (fonctionnelle, institutionnelle, culturelle, identitaire, politique, économique, etc.) de ces traditions qui, on peut le supposer, entretiennent des rapports davantage concurrentiels ou conflictuels qu'harmonieux, en raison des rapports de domination, d'assimilation et de lutte pour la reconnaissance - voire parfois la survie - dont est grevée la traduction au Canada. Il en découlerait une idée plus cohérente et nuancée de cette expérience de la traduction littéraire postulée par Mezei, dès lors appréhendée selon ses facteurs constitutifs et ses lignes de force réelles, d'autant qu'elle est redevable aux manières dont l'ensemble des données bibliographiques énumérées en 2.1 se laissent analyser et interpréter à travers des discours critiques et spéculatifs multiples. 
Chercher à répondre aux questions soulevées par Mezei sans bénéficier d'une vision d'ensemble de la pratique traductionnelle depuis une trentaine d'années ne ferait qu'exposer la contradiction interne, déjà notée, d'un pays officiellement bilingue où la traduction est l'une des pierres angulaires de l'idéologie dominante, que l'on y souscrive ou non. C'est dire que, en dépit de la pertinence, l'intérêt et la richesse des recherches portant sur la traduction littéraire au Canada depuis les années 1980, tout un pan de l'histoire du patrimoine culturel reste difficile d'accès et, partant, constitue un véritable angle mort de la recherche traductologique qu'il importe de recadrer.

\subsection{Des angles morts de la recherche traductologique}

C'est effectivement cet angle mort de la recherche actuelle qui pose problème. Qu'il favorise un certain discours fédéraliste, qu'il provienne d'un désintérêt indéfectible envers l'«autre» ou qu'il soit symptomatique de contraintes (ressources insuffisantes, inadmissibilité aux concours subventionnaires, données empiriques très éparpillées) imposées aux chercheurs universitaires importe peu du moment où l'on se penche sur les effets, dont plusieurs ont été relevés plus haut et auxquels s'ajoute celui-ci: à force de se référer à des sources lacunaires et ponctuées d'erreurs, le risque de laisser perdurer des stéréotypes culturels ou de prendre des idées reçues pour des évidences demeure élevé. D’où, de nouveau, le danger d'une vision plus ou moins décalée par rapport à la réalité des échanges interculturels et à la logique économique, culturelle et politique qui les informe. À cet égard, les formulations plus ou moins complaisantes affichées sur le site du Conseil des arts devraient nous servir de mise en garde, d'autant que des processus de raccourci et d'occultation peuvent s'immiscer au sein de recherches scientifiques basées sur des données empiriques par trop incomplètes.

À titre d'exemple et en référence à une compilation bibliographique à visée exhaustive de toutes les œuvres de fiction publiées par des auteurs anglophones du Québec entre 1991 et 2013, réalisée à partir des variables énumérées en 2.1, il a été possible d'effectuer une série d'analyses statistiques qui ont produit des résultats parfois tout à fait inattendus (voir Lane-Mercier, à paraître). Ainsi, si d'aucuns ont pu réitérer que ces auteurs sont «invisibles» tant au Québec où la majorité francophone ne les lit pas qu'au Canada anglais où ils éprouvent de la difficulté à se faire publier, les graphiques montrent clairement que non seulement plus de la moitié de leurs œuvres ont été publiées au Canada anglais (bien que la tendance baisse légèrement à partir de 2005 en faveur des maisons d'édition anglophones du Québec), mais qu'un original publié en Ontario ou dans une autre province anglophone a, respectivement, 2,24 et 1,79 plus de chances d'être traduit en français qu'un original publié au Québec. Du coup, l’idée largement répandue de la double aliénation des écrivains anglo-québécois se trouve tant soit peu démystifiée, alors que s'esquissent simultanément un déplacement de leurs allégeances vers les maisons d'édition situées dans la région montréalaise et un regain de vitalité de ces dernières, désormais mieux placées pour concurrencer le pouvoir économique et symbolique des maisons torontoises.

L'intérêt d'établir des bases de données à visée exhaustive d'un corpus restreint (ici 800 œuvres de fiction anglo-québécoises, dont 177 [22\%] existent en version française, parmi lesquelles 98 [54,4\%] ont été subventionnées par le Conseil des arts) réside avant tout dans les prédictions qu'elles autorisent, dont celle de vérifier la 
double hypothèse du présent article et de poser des jalons méthodologiques. Le défi consiste dès lors à poursuivre le travail en y adjoignant des sous-ensembles supplémentaires (d'autres genres, d'autres aires géographiques, d'autres littératures minoritaires) pour peu à peu construire le répertoire envisagé. Car si un sous-ensemble isolé facilite la compréhension de certains phénomènes, son inclusion dans un sousensemble plus important (ici le nombre d'œuvres de fiction anglo-canadiennes traduites) en facilite l'explication. Grâce donc à ce corpus restreint, se dégage une série de problématiques que l'analyse statistique permettrait d'approfondir, accompagnées d'avenues de recherche qui se précisent. Sauf exception, ce ne sont pas les questions en soi qui sont nouvelles, mais bien la possibilité de les reposer en fonction de données empiriques quasi exhaustives.

Aussi un répertoire de toutes les œuvres littéraires canadiennes traduites dans les deux langues officielles depuis 1978 permettrait-il de jeter un éclairage analytique plus systématique sur:

- les agences et les agents responsables du processus de traduction;

- la non-traduction;

- le nombre d'auteurs traduits par rapport au nombre d'œuvres traduites;

- le nombre de traductions par année;

- le nombre d'auteurs traduits et publiés à l'étranger;

- la cotraduction et la coédition;

- la répartition géographique des maisons d'édition, des auteurs et des traducteurs;

- le rapport entre le centre et la périphérie, le majeur et le mineur;

- les délais de traduction;

- l'impact des processus de légitimation;

- le rôle et l'impact des agences subventionnaires;

- l'impact de la réimpression et de la réédition;

- l'impact du nombre d'œuvres publiées par un auteur et ses chances d'être traduit;

- le capital symbolique des écrivains-traducteurs;

- les «affinités traductives» (tel auteur/telle maison d'édition a son traducteur attitré);

- la retraduction;

- les genres privilégiés;

- la «surtraduction» (les auteurs systématiquement traduits) et la «sous-traduction»;

- la présence de tendances/traditions propres aux champs anglo-canadien, acadien, franco-ontarien, franco-manitobain, franco-albertain, québécois et anglo-québécois.

- et la liste demeure ouverte. Par la suite, des thèmes ayant déjà fait l'objet d'études partielles pourraient bénéficier d'analyses élargies dans des perspectives synchronique, diachronique et comparatiste, dont par exemple:

- les normes et conventions de la traduction littéraire au Canada;

- les processus de sélection;

- les processus de production et de distribution;

- les processus de canonisation d'œuvres traduites;

- la réception des œuvres traduites;

- la logique de l'industrie de la traduction et du marché du livre traduit;

- la fonction de la traduction entre cultures non paritaires;

- les stratégies de traduction;

- les images de soi et de l'autre véhiculées par les textes traduits;

- la (non-inclusion d'œuvres traduites dans des programmes scolaires et universitaires. 
La prise en compte systématique de données bibliographiques aurait une incidence considérable sur la recherche traductologique au Canada, tout en favorisant, sur un plan plus général, une réflexion ethnographique sur la collecte de données auprès des institutions et de leurs agents. Ceci aurait l'avantage supplémentaire d'articuler les approches quantitative et qualitative (Gile 2011), trop souvent dissociées, et, par là, de donner des assises méthodologiques autrement solides à la sociologie de la traduction, branche de la traductologie actuellement en pleine expansion.

\subsection{Pour une définition de "tradition de traduction"}

L'idée d'une «specific Canadian translation experience» lancée par Mezei en 2003 fait écho à celle d'une "tradition de traduction littéraire» énoncée par Stratford en 1977. En opposant au constat négatif de Stratford l'éventualité d'une telle tradition, l'interrogation de Mezei contient en germe ma deuxième hypothèse. En effet, tout porte à croire que l'accélération des processus d'autonomisation des institutions littéraires anglo-canadienne, québécoise, franco-ontarienne et acadienne, l'augmentation du nombre d'œuvres littéraires traduites, de même que la consécration du traducteur comme créateur au même titre que l'auteur qui ont marqué les années 1970 et 1980 sont autant d'indices que le terrain était enfin propice à l'éclosion de véritables lignes de force traductives. Mentionnons également le nombre croissant d'études traductologiques qui ont relativisé le constat de Stratford, de plus en plus difficile à justifier. Plusieurs iront jusqu'à revendiquer, tantôt à partir de pratiques traductives marginales, dont la traduction «au féminin» (Simon 1996; von Flotow 1997) et la traduction «déviante» (Simon 1994, 2000), tantôt à partir d'études de cas spécifiques (Whitfield 2005; 2006; Koustas 1997; 1998; à paraître), la présence d'une tradition canadienne-anglaise ou québécoise, sans toujours la définir. Si donc il manque sur le plan empirique un répertoire exhaustif, il manque sur le plan conceptuel une définition claire de «tradition de traduction", à fortiori lorsqu'il s'agit de la concevoir au pluriel.

Bien qu'il ne soit pas encore possible de vérifier notre deuxième hypothèse, on peut en revanche amorcer une réflexion sur l'idée d'une coexistence conflictuelle et mouvante de plusieurs traditions depuis une trentaine d'années, lesquelles confèrent à la traduction littéraire canadienne tant sa dynamique que ses configurations propres. La définition proposée par Stratford en 1977 offre un bon point de départ en vertu de la perspective institutionnelle qui la sous-tend. Selon lui, «tradition de traduction» signifie:

(a) une volonté de systématiquement traduire les œuvres littéraires dans l'autre langue officielle;

(b) la mise en place de politiques traductionnelles;

(c) la création d'une infrastructure subventionnaire (voir Stratford 1977: i-viii);

éléments qui selon lui n'étaient qu'embryonnaires à l'époque. Dans un deuxième temps cependant, il s'agit d'élargir cette définition à la lumière des données concrètes dont nous disposons depuis la fin des années 1970. Aussi deux réorientations s'imposentelles. 


\subsubsection{Première réorientation de la définition}

Même incomplètes, les données disponibles suffisent à montrer que la structure décrite par Stratford ne s'est jamais concrétisée. D’une part, les œuvres ne sont toujours pas systématiquement traduites dans l'autre langue (Koustas 1998) et l'écart entre traduction et non-traduction se creuse ${ }^{14}$ malgré l'injection de sommes importantes par le gouvernement fédéral en 2009. D’autre part, plus solide de nos jours qu'en 1977, l'infrastructure subventionnaire comporte d'importants déséquilibres si l'on s'en tient à son mandat explicite, axé pourtant sur l'égalité. Ainsi, si entre 1972 et 1992 le Conseil a subventionné 581 traductions vers le français et 585 traductions vers l'anglais, derrière la symétrie se cachent des variations tant sur le plan des montants accordés (329 000 \$ vers l'anglais; 255000 \$ vers le français) qu'à celui des genres littéraires traduits, le Québec préférant traduire des œuvres de non-fiction et le Canada anglais des œuvres de fiction (Ellenwood 1983). Qui plus est, entre 1991 et 2013, la symétrie numérique disparaît complètement en faveur de la traduction vers le français : 1036 œuvres traduites contre 765 vers l'anglais. Doit-on en conclure qu'il n'existe toujours pas de tradition de traduction littéraire au Canada?

Bien au contraire, cette persistance dans le déséquilibre signale à la fois une «asymétrie systématique» et, du coup, la présence de traditions traductives francophones et anglophones dissemblables aux contours fluctuants mais distincts. Il faudrait par conséquent remettre en question - voici la première réorientation de la définition de «tradition» qui s'impose - moins la perspective institutionnelle de Stratford que la pertinence d'une définition qui masque les fluctuations et inégalités, afin d'éviter une tendance qui, conformément au discours fédéral sur le bilinguisme officiel, consiste à privilégier la parité au détriment des tensions, rapports de force et disparités sousjacents. Cela dit, l'absence d'un tableau d'ensemble, comprenant entre autres le nombre de traductions non subventionnées et le nombre de non-traductions, nous empêche de saisir les enjeux (politiques, institutionnels, socioculturels, économiques, etc.) précis de ces traditions. Nous ne pouvons que rester sur le plan hypothétique en réitérant l'observation du traducteur anglo-canadien Larry Shouldice: «I am inclined to think that the major political effect of literary translation in Canada has been to make articulate our differences» (1983: 82) ou encore se reporter aux analyses statistiques faites à partir de la bibliographie de Stratford qui commencent à dater...

\subsubsection{Deuxième réorientation de la définition}

À la décharge de Stratford qui ne possédait pas le recul que nous avons aujourd'hui, son "Introduction" (i-viii) à la Bibliographie de livres canadiens traduits de l'anglais au français et du français à l'anglais (Stratford 1997) offre des pistes d'analyse quantitative et qualitative qui préfigurent l'élargissement de sa définition de «tradition ». Après avoir recensé quelque 640 titres depuis la colonisation du Canada, il procède à des comparaisons d'ordre statistique (nombre d'œuvres traduites vers chacune des langues officielles; progression au fil des années; genres privilégiés), pour ensuite esquisser le type d'analyses auxquelles ces comparaisons ouvrent la voie. Ainsi, remarquant que, au cours de la période observée, les traductions du français à l'anglais ont toujours dépassé celles effectuées dans l'autre sens, il pose les jalons d'interprétations d'inspiration sociohistorique (existence de certains mythes et blocages concernant l'«autre»), éditoriale (absence d'une infrastructure adéquate), 
économique (la traduction littéraire est mal payée), géographique (rôle des éditeurs et traducteurs européens) et culturelle (l'histoire du Québec est plus accessible en français qu'en anglais).

D’après Koustas, l'une des rares traductologues canadiennes qui a tenté d'élucider la notion de tradition, "a study of the tradition of translation practice entails a broader consideration not only of the methods used but of both the translators' and publishers' selection of the works to be translated» (1997: 44). En effet, ce qui fait défaut à la définition de Stratford, outre la notion de la traduction comme pratique signifiante, c'est une articulation explicite entre le social (ou l'institutionnel) et l'individu, d'un côté, et, de l'autre, entre le social et l'histoire, toute tradition étant issue d'un passé que l'on cherche à perpétuer en le transmettant de génération en génération, tel un héritage. Dans cette optique, peuvent se constituer en traditions aussi bien des processus, tels que des actions, des pratiques, des façons de faire et de penser, que des produits, tels que des doctrines, des croyances, des informations, des savoirs et des textes qui acquièrent le statut d'habitudes ou de coutumes à transmettre au sein d'un groupe sur une durée relativement longue.

Il en ressort que si l'on peut concevoir une histoire dépourvue de traditions, à l'instar de l'histoire de la traduction littéraire au Canada jusqu'à la fin des années 1970, l'inverse est impossible, toute tradition présupposant un rapport entre le passé et le présent, ainsi que des agents et des agences susceptibles de la transmettre en fonction de normes, conventions et attentes préétablies. De même que les traditions sont susceptibles d'évoluer, voire se transformer, se fragmenter, décliner ou disparaître, de même elles peuvent se heurter à des traditions parallèles plus ou moins antagonistes ou encore à des résistances internes génératrices de contre-traditions; phénomènes qui vont à l'encontre d'une vision monolithique de l'idée de tradition qui guette aussi bien la définition de Stratford que l'idéologie pancanadienne, lesquelles se réfèrent à «une» tradition de traduction littéraire au Canada et à «une» littérature canadienne, respectivement.

En avançant la présence de traditions traductives non coïncidentes, notre deuxième hypothèse revêt à son tour une dimension programmatique pour autant qu'elle pointe vers la nécessité de forger un appareil conceptuel à partir duquel il sera possible non seulement de les définir, mais aussi de les identifier et de les analyser. Ce n'est qu'à ce prix que nous serons en mesure de répondre autrement que par déductions à ces deux questions clés soulevées par Mezei (2003: 107) il y a dix ans et qu'il vaut la peine de répéter: "How do we stake out a history of literary translation in Canada?» et, surtout, «Is there a specific Canadian translation experience?».

\subsection{Vers de nouvelles questions méthodologiques}

À supposer que les deux carences soient comblées, c'est-à-dire que nous disposions d'un répertoire exhaustif des traductions littéraires depuis 1978 et d'une acception limpide de "tradition de traduction littéraire», resterait à trouver des réponses à des questions d'ordre méthodologique. Comment faire l'articulation entre l'analyse quantitative et l'analyse qualitative, d'un côté, et entre l'analyse sociologique et l'analyse traductologique (ou textuelle, souvent négligée par les sociologues), de l'autre? À partir de quel moment une tendance se transforme-t-elle en tradition? Comment en suivre l'émergence, l'évolution et, éventuellement, l'affaiblissement? Comment 
procéder à la comparaison de traditions disparates, voire antagonistes? Car en postulant que par «tradition" il faut entendre la mise en phase de constantes, de systématicités, de complexes, d'habitudes, de normes qui se transmettent, sous forme de processus et de produits, d'un groupe social ou professionnel à un autre par le truchement d'agents et d'agences déterminés, encore faut-il forger, outre un appareil conceptuel, un appareil méthodologique apte à les appréhender.

\subsubsection{Premier exemple: la traduction vers l'anglais du joual littéraire}

J'aimerais donner deux exemples qui permettront d'ajouter des éléments définitionnels supplémentaires à ceux déjà convoqués, tout en posant quelques assises méthodologiques qu'il faudrait bien entendu consolider par la suite. Ces exemples renvoient à des problématiques déjà amplement explorées et souvent citées lorsqu'il est question d'une «école canadienne» de la traduction, lesquelles auraient néanmoins tout avantage à être approfondies, élargies ou généralisées à la lumière de données empiriques plus complètes et à jour.

Le premier exemple a trait aux problèmes esthétiques, historiques, sociopolitiques et identitaires soulevés, en contexte canadien, par la traduction vers l'anglais des québécismes en général et du joual ${ }^{15}$ en particulier, où les anglicismes abondent. Comme l'a démontré Sherry Simon (1988), les traducteurs littéraires anglo-canadiens ont eu pendant longtemps recours à des stratégies traduisantes, sinon aléatoires, du moins variées. Or, il y a fort à parier que, à force d'être mobilisées en fonction d'impératifs de plus en plus consensuels liés à l'apparition au sein de la culture cible (le Canada anglais) de normes et d'attentes fondées, notamment, sur la lisibilité et l'acceptabilité, ces stratégies se sont «normalisées » pour donner lieu, dans le courant des années 1970 et 1980, à ce qui pourrait ressembler à une tradition de traduction de la québécitude ou, plus modestement, du joual.

Autrement dit et en se limitant au cas du joual, il se serait produit aux alentours de 1980 une convergence complexe de processus, agents, agences et textes relativement stables (des traducteurs prêts à s'attaquer à ce genre de problème traductionnel; des éditeurs prêts à publier des traductions à teneur nationaliste, voire souverainiste; un corpus d'œuvres à traduire; un ensemble cohérent de stratégies de traduction; des pratiques éditoriales de révision et de promotion; des modalités de réception et de consécration) que l'on aurait cherché à perpétuer. Voici, précisément, ce qu'une compilation complète d'œuvres joualisantes traduites en anglais permettrait de vérifier (ou non), car les études partielles menées jusqu'ici indiquent que, à partir du début des années 1970, le joual a eu tendance à être rendu par des stratégies d'assimilation visant à évacuer le projet identitaire et politique pour un public canadienanglais hostile au nationalisme québécois.

De façon peut-être encore plus significative, en révélant, du coup, le nombre d'œuvres joualisantes non traduites, une telle compilation rendrait possibles, en plus de la question incontournable de savoir pourquoi (censure? difficultés traductionnelles trop élevées? mauvaise réception des œuvres joualisantes déjà traduites?), des analyses dorénavant plus rigoureuses de certaines facettes de l'activité traduisante symptomatiques d'une tradition au Canada anglais. Ces analyses révéleraient, entre autres, la «logique» - jusqu'ici difficile à saisir sur le double plan synchronique et diachronique - qui informe depuis une cinquantaine d'années les critères de sélection comme les stratégies traductionnelles d'œuvres joualisantes. 


\subsubsection{Deuxième exemple: la traduction «au féminin»}

Le deuxième exemple a trait à la traduction «au féminin », souvent qualifiée de typiquement canadienne d'autant qu'elle est indissociable de pratiques d'écriture expérimentales développées au Québec par des écrivaines féministes au cours des années 1970 et 1980. Celles-ci ont alimenté des pratiques traduisantes tout aussi expérimentales chez des traductrices anglo-québécoises et canadiennes à l'intention d'un lectorat initialement canadien-anglais. Dans sa préface à Translating Women (2011), Luise von Flotow retrace l'émergence et l'évolution de ce qu'elle appelle, avec le recul, le premier paradigme de la traduction au féminin. Ce faisant, elle met en place, ne serait-ce que schématiquement, les fondements méthodologiques généraux pour l'identification et l'analyse, à partir de données empiriques, de toute tradition de traduction littéraire, en fonction des divers paramètres de nature esthétique, sociale, historique, institutionnelle et politique énumérés plus haut.

En effet, ce qui domine la préface de von Flotow, c'est le rapport passé-présent si central à l'idée de tradition:

It is time to write about «women and translation» again, time to return to and perhaps expand on the «first paradigm» of gender studies as applied to translation, revisiting a series of agents [...]. Inevitably, in returning here, feminist social and cultural activism is brought back into play. Almost twenty years have passed since the first work [...] started to appear in North America [...]. (von Flotow 2011: 1)

Suit une série chronologique de repères qui, en s'accumulant, représentent autant d'étapes dans la consolidation d'une pratique à transmettre, avec ses agents, ses agences, ses textes et ses processus propres, à savoir, dans un premier temps, le corpus d'œuvres à traduire en anglais, les stratégies traduisantes déployées et les essais rédigés par les traductrices, lesquels ont inspiré, dans un deuxième temps, des articles et monographies théoriques et historiques plus scientifiques consacrés à l'apport des diverses formes de féminisme à la traduction et à la traductologie. Comme le précise von Flotow, « [t]his work, largely by women researchers, translators, and editors, [...] collected and distilled, and doubtless constructed, women's «voices» in translation $[\ldots] »(2013: 2)$.

Si elle insiste de prime abord sur la dimension internationale de la traduction au féminin, toujours est-il que les nombreuses références à des traductrices et traductologues canadiennes soulignent le rôle que celles-ci ont joué, à commencer par von Flotow elle-même. Se dessinent alors implicitement les contours d'une expérience et d'une tradition de traduction au féminin plus spécifiquement canadiennes, avec les variables qui les jalonnent et la manière de les identifier et de les analyser. Fait enviable à noter au passage: le caractère marginal (et marginalisé) de cette tradition au Québec et au Canada anglais a rendu relativement aisées les compilations bibliographiques tant soit peu exhaustives, de sorte que les analyses peuvent se fonder sur des chiffres et des statistiques essentiellement à jour.

La préface de von Flotow a l'énorme mérite de présenter un aperçu évolutif d'une tradition qui s'est transformée au fil des années. Si, à l'heure actuelle, certains savoirs, façons de penser et pratiques inhérents au premier paradigme continuent à être transmis, d'autres ont cédé la place à de nouveaux savoirs et pratiques qui, selon elle, pourraient former à la longue les lignes de force d'un deuxième paradigme axé non plus sur la notion de féminin mais bien sur celle de performativité, issue des queer 
theories. Il me semble que l'essor et l'éventuel renouveau d'une tradition canadienne de traduction au féminin, pour expérimentale et circonscrite qu'elle soit au sein du champ, pourraient servir de prototype à l'analyse d'autres traditions ayant elles aussi émergé depuis 1980, à la condition toujours d'avoir accès à un répertoire complet et à jour des œuvres littéraires traduites à partir de et dans les deux langues officielles du Canada.

\section{Conclusion}

En guise de conclusion, j'aimerais souligner le caractère instructif des deux carences que j'ai voulu mettre au jour, en ce qu'elles nous révèlent l'ampleur du travail qu'il reste à faire, que ce soit au Canada ou dans tout pays officiellement bi- ou multilingue ne disposant pas d'un répertoire bibliographique fiable des œuvres littéraires traduites dans la ou les autres langues officielles. Pour ce qui est de la conjoncture canadienne, non seulement l'analyse quantitative (statistique) et qualificative (interprétative) conjuguée rendrait enfin possibles l'identification, l'analyse, la conceptualisation et la comparaison, jusqu'ici élusives, de plusieurs traditions traductives non coïncidentes, mais, plus largement, elle représenterait une contribution concrète et originale à l'avancement de la recherche dans le domaine de l'histoire de la traduction littéraire au Canada comme dans celui de l'histoire culturelle et littéraire canadienne comparée. En se rappelant à la suite de Sapiro que les chiffres ne sont jamais neutres, l'établissement d'une Bibliographie d'œuvres littéraires canadiennes traduites de l'anglais au français et du français à l'anglais depuis 1978 faisant suite à celle de Stratford permettrait de jeter un regard aussi stimulant qu'inédit sur l'évolution du rapport entre l'individuel, le culturel et le social au cours des trente dernières années, ainsi que sur le rôle social du traducteur et celui de la traduction. Ce faisant, une bibliographie mettrait clairement au jour une troisième carence - et non des moindres -, soit l'exclusion des traductions littéraires dans les langues non officielles, à commencer par les langues autochtones, historiquement refoulées; carence sousjacente à l'ensemble des prémisses de cette étude et, de manière sans doute bien plus insidieuse, à la loi sur les langues officielles du Canada. Voilà un effet autrement instructif du répertoire tel qu'il a été conçu ici.

Or, il va de soi que les "silences» bibliographiques ne sont pas uniques au contexte canadien et que les questions soulevées tout au long du présent article auraient besoin d'être à leur tour élargies afin de prendre la mesure des enjeux qu'elles posent à une échelle plus globale, notamment (mais pas exclusivement) dans les pays postcoloniaux où les rapports de force entre langues dominante(s) et dominées ont souvent été exacerbés par la mise en place de politiques linguistiques nationales. La mise au jour de traditions conflictuelles de traduction littéraire dans les pays officiellement bi- ou multilingues pourrait influer, d'une part, sur l'étude comparée - tant intranationale que transnationale - de politiques culturelles et, d'autre part, sur l'appréciation de l'évolution des identités culturelles linguistiques fondées moins sur le propre que sur la «relationalité» et la diversité.

Dans une perspective traductologique, enfin, le concept de «traditions de traduction» me paraît particulièrement porteur et mériterait d'être réexaminé en fonction d'autres contextes. Comme le notait Edwin Gentzler, «the complicated question of Canadian identity - problems of colonialism, bi-lingualism, nationalism, 
cultural heritage, weak literary system, and gender issues are involved - seems to provide a useful platform from which to begin raising questions about current translation theory» (1993: 184). Aussi l'approche intégrée systématique laisse-t-elle présager une meilleure articulation des approches sociologiques tant à l'historiographie de la traduction littéraire qu'à l'analyse textuelle de traductions (Wolf et Fukari 2007). Allons encore plus loin: l'établissement de tels répertoires pourrait avoir un impact direct dans la conjoncture de mondialisation actuelle où les définitions officielles du multiculturalisme et de la diversité linguistique sont en pleine mutation.

\section{NOTES}

1. Par «institutionnel», j’entends souligner le rôle, incontournable dans les universités canadiennes, de l'appui financier des agences subventionnaires fédérales et provinciales, sans lequel il est pour ainsi dire impossible de mener à terme une recherche scientifique d'envergure.

2. Si la traduction joue un rôle depuis la découverte du Canada par Jacques Cartier, la traduction littéraire sera assez marginale jusqu'aux années 1960. À titre d'indication, avant 1960, seulement 16 romans furent traduits de l'anglais au français et 39 du français à l'anglais (Stratford 1997: iv).

3. Sans entrer dans l'histoire de l'édition littéraire au Canada, précisions que l'établissement d'un tel répertoire présuppose a) que l'on ait une définition claire de qui peut être qualifié d'« auteur canadien » et b) que l'on ait décidé d'y inclure (ou exclure) les œuvres originales publiées à l'étranger (Angleterre, États-Unis) ainsi que les œuvres traduites publiées à l'étranger (France, Belgique).

4. Conseil des arts du Canada. (Dernière mise à jour: 2 juin 2005), http://conseildesarts.ca/cgibin/MsmGo.exe?grab_id=0\&page_id=2500\&query=traduction\&hiword=TRADUCTEUR\%20 TRADUCTEURS\%20TRADUCTIONS\%20TRADUCTRICE\%20traduction\%20

5. Bibliothèque et archives Canada. (Dernière mise à jour: 16 novembre 2004), http://amicus.collectionscanada.gc.ca/aaweb/aaloginf.htm

6. Par «droit fondamental», on entend celui qui consiste à permettre à chaque citoyen canadien d'obtenir les services offerts par le gouvernement fédéral dans la langue officielle de son choix. La présence quotidienne du bilinguisme officiel est assurée, entre autres, par l'affichage bilingue obligatoire des espaces fédéraux (ex. les aéroports) et l'étiquetage des produits de consommation.

7. Ici encore, il importe de relativiser. Plusieurs bibliographies partielles ont été établies par des traductologues canadiens au cours des vingt dernières années, dont celle de G. Nichols qui recense toutes les traductions d'œuvres dramatiques canadiennes et étrangères pour la scène locale et internationale entre 1900 et 2000 (Nichols 2001; 2002). Cela dit, ces répertoires partiels, difficiles à entretenir faute de fonds, sont rarement publiés ou mis en ligne.

8. Plus spécifiquement, le pancanadianisme prôné par le discours fédéraliste conçoit le Canada comme «une communauté politique purement civique, bilingue, multiculturelle et uninationale, fondée sur une appartenance à des valeurs telles que l'égalité juridique absolue, la symétrie entre les provinces, la tolérance à l'endroit des différences religieuses et culturelles exprimées (uniquement) dans la sphère privée et l'inaliénabilité des droits individuels [...]. Les habitants [...] n'étant pas d'un genre différent, devraient jouir des mêmes droits et, ultimement, se représenter d'abord et avant tout comme Canadiens» (Maclure 2000: 149). C'est dire que le pancanadianisme refuse de reconnaître non seulement l'idée de la diversité culturelle, mais également les revendications communautaires ou nationalistes des minorités ethniques, culturelles ou linguistiques (Caron 2013).

9. Conseil des arts du Canada. (Dernière mise à jour: 18 novembre 2009.) Consulté le 31 mai 2013, www.conseildesarts.ca/NR/exeres/7ED3EFF4-E357-47A6-9AA7-18148642CBF2.htm

10. Conseil des arts du Canada. (Dernière mise à jour: 20 janvier 2010.) Consulté le 31 mai 2013, www. conseildesarts.ca/cgi-bin/MsmGo.exe?grab_id=0\&page_id=5307\&query=traducteur\&hiword=TR ADUCTEURS\%20TRADUCTION\%20TRADUCTIONS\%20TRADUCTRICE\%20traducteur\%20

11. Conseil des arts du Canada. (Dernière mise à jour: 3 mai 2010.) Consulté le 31 mai 2013, www. conseildesarts.ca/cgi-bin/MsmGo.exe?grab_id=0\&page_id=5310\&query=traducteur\&hiword=TR ADUCTEURS\%20TRADUCTION\%20TRADUCTIONS\%20TRADUCTRICE\%20traducteur\%20

12. Conseil des arts du Canada. (Dernière mise à jour: 29 mai 2008.) Consulté le 31 mai 2013, www. conseildesarts.ca/aproposdenous/Contexte/ry128565584923495235.htm. Ces valeurs ont elles aussi une histoire: «Le Conseil estime que les neuf valeurs énumérées ci-dessous sont ses valeurs fon- 
damentales. Ces valeurs ont évolué au cours de l'histoire du Conseil, et plusieurs d'entre elles sont apparues dans les premières années de son existence.» L'intérêt pour les langues et cultures autochtones est récent.

13. Voir, respectivement, www.dcu.ie/ ctts et www.ttle.satd.uma.es

14. Ceci est une déduction, non encore vérifiable, faite à partir du corpus d'œuvres anglo-québécoises, où l'écart se creuse de manière spectaculaire depuis 2007.

15. Le joual est un parler populaire des quartiers ouvriers francophones de Montréal, dont les caractéristiques linguistiques principales sont la présence très élevée d’anglicismes, un lexique appauvri, surchargé de «sacres» et de blasphèmes, une syntaxe déviante et une prononciation non standard. Des représentations du joual ont commencé à apparaître dans le roman, la poésie et le théâtre québécois au début des années 1960 à des fins politiques (nationalistes) et identitaires, en vue de récuser simultanément la domination économique du Canada anglais et la domination culturelle de la France.

\section{RÉFÉRENCES}

BAssnet, Susan (1993): Comparative Literature: A Critical Introduction. Oxford: Blackwell.

Beaudoin, Réjean et Lamontagne, André (2002): Un demi-siècle de réception critique de la littérature québécoise au Canada anglais: 1939-1989. Canadian Literature. 27:19-43.

BRISSET, Annie (1990): Sociocritique de la traduction: théâtre et altérité au Québec (1968-1988). Longueuil: Le Préambule.

CARon, Jean-François (2013): L'évolution du fédéralisme canadien et son incidence possible sur le discours sécessionniste au Québec. L'actualité fédérale. 4(4):1-8.

Chevrel, Yves, D’hulst, Lieven et Lombez, Christine (dir.) (2012): Histoire des traductions en langue française. Dix-neuvième siècle (1815-1914). Paris: Éditions Verdier.

Delisle, Jean (1987): La traduction au Canada/Translation in Canada: 1534-1984. Ottawa: Presses de l'Université d'Ottawa.

Ellenwood, Ray (1983): Some Actualities of Canadian Literary Translation. In: Camille LA Bossıère (dir.) Translation in Canadian Literature. Ottawa: Ottawa University Press, 61-71.

Ellenwood, Ray (1984): Some Notes on the Politics of Translation. Atkinson Review of Canadian Studies. 2(4):25-28.

GentzLer, Edwin (1993): Contemporary Translation Theories. London: Routledge.

GiguÈre, Richard (1983): Traduction littéraire et «image» de la littérature au Canada et au Québec. In: Camille LA Bossıère (dir.), Translation in Canadian Literature. Ottawa: Ottawa University Press, 47-60.

GiLE, Daniel (2011): La recherche traductologique: méthodes ou approches? TTR. 24(2):41-64.

Godbout, Patricia (2004): Traduction littéraire et sociabilité interculturelle au Canada 1950-1960. Ottawa: Les Presses de l'Université d'Ottawa.

Hayne, David (1983): Literary Translation in Nineteenth-Century Canada. In: Camille LA Bossière (dir.), Translation in Canadian Literature. Ottawa: Ottawa University Press, 35-46.

Koustas, Jane (1997): Quebec Literature in Translation: Loaded Canons. Québec Studies. 23: 43-53.

Koustas, Jane (1998): Lost from the Canon: The Canada Council and French-English Translation since 1979. Gerstein Research Seminar, Les Études traductologiques au Canada. Toronto: Glendon College (inédit).

Koustas, Jane (2008): Les Belles Etrangères: Canadians in Paris. Ottawa: University of Ottawa Press.

Koustas, Jane (à paraître): Translating Canada: From Dichotomy to Diversity.

LANE-Mercier, Gillian (2011) : Éléments pour un nouveau paradigme des rapports de contiguïté socioculturelle et disciplinaire. L'exemple du Canada et du Québec. In: Francis Mus, Karen Vandemeulebroucke, Lieven D'hulst et Reine Meylaerts (dir.), La traduction dans les cultures plurilingues. Arras: Artois Presses Université, 161-172.

LANE-MERCIER, Gillian (à paraître) : La fiction anglo-québécoise en traduction française depuis 1990: agents, agences et textes. Recherches sociographiques. 
Linteau, Paul (1996): Traduire l'histoire du Canada 1975-1996. Études canadiennes/Canadian Studies. 41:87-98.

Lotbiniere-Harwood, Susanne de (1991): Re-belle et infidèle: la Traduction comme pratique de réécriture au féminin/The Body Bilingual: Translation as a Rewriting in the Feminine. Montréal et Toronto: Les éditions du remue-ménage et Women's Press.

Maclure, Jocelyn (2000): Récits identitaires. Le Québec à l'épreuve du pluralisme. Montréal: Éditions Québec Amérique.

Mezei, Kathy (2003): Dialogue and Contemporary Literary Translation? In: Denis SaintJACQUes (dir.), Tendances actuelles en histoire littéraire canadienne. Sainte-Foy: Nota bene, 107-129.

Mezei, Kathy, Simon, Sherry et von Flotow, Luise (dir.) (2014): Translation Effects: The Shaping of Contemporary Canadian Culture. Montréal: McGill-Queen's University Press.

Munday, Jeremy (2001): Introducing Translation Studies: Theories and Applications. London: Routledge.

Nichols, Glen (2001): From Around the World and at Home: Translation and Adaptation in Canadian Theatre. Toronto: Playwrights Union of Canada.

Nichols, Glen (2002): Trading Partners: New Views on Theatre Translation in Canada. TTR. 15(1):117-135.

SAPIRO, Gisèle (dir.) (2008): Translatio: le marché de la traduction en France à l'heure de la mondialisation. Paris: CNRS Éditions.

Simon, Sherry (1988): The True Quebec as Revealed to English Canada: Translated Novels 1864-1960. Canadian Literature. 117(1):31-43.

Simon, Sherry (1994): Le trafic des langues. Montréal: Boréal.

Simon, Sherry (1996): Gender in Translation: Cultural Identity and the Politics of Transmission. London: Routledge.

Simon, Sherry (1997): Translation and Cultural Politics in Canada: Post Colonial Contexts. In: Shantha Ramakrishna (dir.), Translation and Multiculturalism. Delhi: Pencroft International, 83-95.

Simon, Sherry (2000): Pratiques déviantes de la traduction. Francophonies d'Amérique. 10:159-166.

Simon Sherry (2006): Translating Montreal: Episodes in the Life of a Divided City. Montréal: McGill-Queens University Press.

St-Pierre, Paul (1993): Translation as a Discourse of History. TTR. 6(1):61-82.

Stratford, Philip et Newman, Maureen (1977): Bibliography of Canadian Books in Translation: French to English and English to French /Bibliographie de livres canadiens traduits de l'anglais au français et du français à l'anglais. $2^{\mathrm{e}}$ éd. Ottawa: CCRH.

von Flotow, Luise (1997): Translation and Gender. Translation in the "Era of Feminism". Manchester and Ottawa: St. Jerome Publishing and University of Ottawa Press.

von Flotow, Luise (dir.) (2011): Translating Women. Ottawa: University of Ottawa Press.

Whitfield, Agnes (dir.) (2005): Le métier du traducteur. Portraits de traducteurs et traductrices littéraires de la francophonie canadienne et québécoise. Montréal: Fides.

Whitfield, Agnes (dir.) (2006): Writing Between the Lines. Portraits of Canadian Anglophone Translators. Waterloo: Wilfred Laurier University Press.

Wolf, Michaela et Fukari, Alexandra (dir.) (2007): Constructing a Sociology of Translation. Amsterdam: Benjamins. 